\title{
An Efficient Home Automation Approach using Raspberry Pi in Wireless Sensors with Smart Phone
}

\author{
Sunil Kumar \\ M. Tech Scholar \\ Uttarakhand Technical University \\ Dehradun, India
}

\author{
Astha Sharma \\ Assistant Professor \\ Uttarakhand Technical University \\ Dehradun, India
}

\begin{abstract}
Home automation is an important milestone that is becoming increasingly prominent day by day because of its various points of interest. Advancement in existing technologies have made smart homes that are more efficient and secure. This can be done either by local system administration or through remote control. Using the Raspberry Pi in home automation provides various features to turn a regular home into a smart home. Raspberry $\mathrm{Pi}$ is a small computer, which was presented in the year of 2012. it is at present a standard framework subject to broad accessibility that can be utilized as a part of home automation. Raspberry Pi provides a low cost platform for interconnecting electrical/electronic devices and various sensors in a home via the internet network. The main objective of present work is to design a smart home which can be controlled and monitored by the Raspberry $\mathrm{Pi}$ via the Internet of Things (IoT). This will help the home owners to provide a simple, fast and reliable way to automate their environment. This paper shows an ease and adaptable smart home automation framework utilizing Raspberry Pi, with IP availability for getting to and controlling appliances and remotely utilizing Smart telephone application. In proposed framework for designing interface we are using PHP, coding is done in Python, WiringPi is used in Raspberry $\mathrm{Pi}$ as a library.
\end{abstract}

\section{Keywords}

Home Automation, Internet of Things, Raspberry Pi, Printed Circuit Board

\section{INTRODUCTION}

The Internet of Things (IoT) can be depicted as interfacing regular articles like advanced mobile phones, InternetTVs, sensors and effectors to the Internet where the equipments are properly connected together empowering new types of correspondence amongst things and individuals, and between things themselves. Building IoT has progressed essentially over the most recent a long time since it has added another measurement to the universe of data and correspondence advancements. Home automation or Smart Homes can be depicted as presentation of innovation inside the home condition to give accommodation, solace, security and vitality productivity toits users. Adding knowledge to home condition can give expanded personal satisfaction. With the presentation of the Internet of Things (IoT), the examination and usage of home automation are getting more famous.

It is method used for equipments which sense physical events then make an interpretation of them into a flood of data, information, expands wellbeing, security, comfort and vitality reserve funds. The building components of home automation are sensor hubs and Building Administration System (BMS). Sensor Web components, it's utilized as a piece of it, moving a BMS to worldwide level. Along these lines, home computerization can be characterized as an instrument expelling human cooperation as much as actually conceivable and attractive in different residential procedures and supplanting them with modified electronic frameworks.

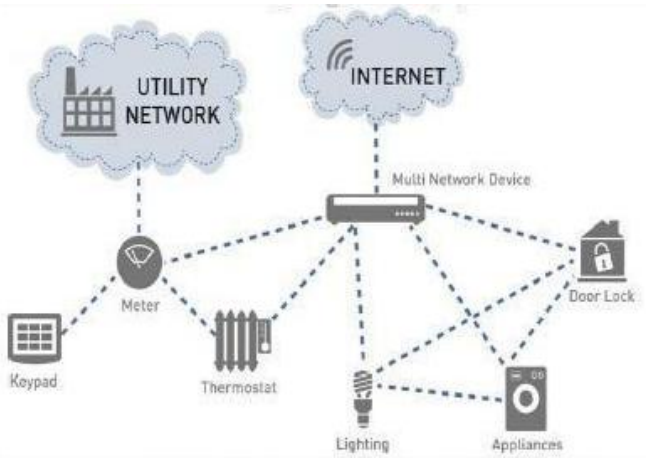

Fig 1: Home Appliances connected to Network [1]

\section{LITERATURE SURVEY}

Smart home is not a new term for science society, it is been utilized from decades. As electronic advances are growing, the field of home automation is extending fastly. There were different existing frameworks have been proposed where the control is by means of Bluetooth, web and so forth. Bluetooth capacities are great and the vast majority of current portable workstation/desktops, tablets, scratch pad and PDAs have worked in connector to decrease the cost of the framework. In Wi-Fi based home automation framework is displayed. It utilizes a PC (with worked in Wi-Fi card) based web server that deals with the associated home gadgets. The framework underpins an extensive variety of home automation equipments like fans, lights, other home machines.

This paper intends to expand on the past research portrayed to actualize a remote sensor system to screen machines in the house. These apparatuses will be controlled through a cell phone running Android OS. This approach gives a simple to work and financially savvy approach that will profit clients to interface with Home machines remotely[1].

This paper gives the complete overview of a wireless home automation system (WHAS) which has been built and implemented according to users need. The automation focuses on voice recognition commands and uses low-power RF ZigBee wireless communication modules which are relatively cheap. The home automation system is intended to control all electrical appliances in a home using voice commands. The paper does however fortify the benefits of utilizing a remote standard. Bluetooth is a worldwide standard for interfacing an extensive variety of gadgets, it is accessible on most handheld gadgets, the innovation is anything but difficult to utilize and set up, and it gives security by encoding information utilizing a 128bit since quite a while ago shared key yet the 
inconvenience of framework is that it has a short scope of correspondence[4].

\section{METHODOLOGY}

The methodology of my thesis is based on a technique named as Raspberry PI and coding is done in PYTHON language.

Brief introduction of technologies is given below.

\subsection{Basic Structure of Proposed work}

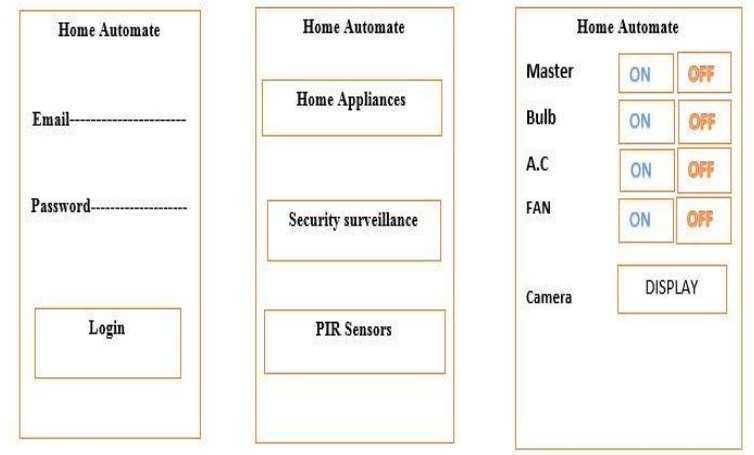

Fig 2: Basic Structure of Proposed Work

This Figure gives the basic structure of the proposed method through which we show the sample layout of the proposed method.

\subsection{Basic elements of proposed work Interface}

Once the layout was created, it was time coordinate all the improvement work to make an entire interface. The python code deals with the rundown of controls that are to be shown to the client. The product bundle gives different perspectives to control diverse parts of the controls, for example, how they are overseen and how they show up on particular customer programming. The Servlet application keeps running on Open Shift PAAS wherein the Raspberry Pi microchip and the clients will be customers to it. Since Open Shift0 can give all the more intense applications, a more propelled UI was made.

\section{Software}

The server and customer application is composed utilizing Python code as the interface for Raspberry Pi to associate with lights and sensors. The product delivered uses mainly following softwares

- $\quad$ SD Formatter

- WIN 32 Image Writer

- WinSCP-Server

\section{Programming Language}

We are mainly using three programming languages which are as follows:

- $\quad$ HTML

- PYTHON

- $\quad$ PHP

\section{Hardware}

The following stage was the reconciliation of the electronic segments into our Raspberry Pi and setting it up for remote get to. Here we utilize a D-Link $2750 u$ switch that has an ISP association. The equipment's collaboration happens through Wi-Fi wherein all gadgets are associated with the Raspberry
Pi through Ethernet. The Android Application Controls the Integrated peripherals, for example, Camera, Door Sensors and Lighting Devices.

The gadgets created empower the client to control the machines utilizing prior gadgets, for example, their Smartphone or home PC. The interfaces are instinctive and simple to utilize and give the client a more available interface then those found in the home. The gadgets are additionally simple to coordinate into existing applications and require just a little measure of mastery to introduce. In proposed work I will plan a framework that speaks with a cell phone, for example, a Smartphone or tablet by means of Raspberry Pi to control an entryway sensors and a light switches and a camera to stream live video, yet has numerous conceivable applications that could profit by this work.

\section{Raspberry Pi}

The Raspberry $\mathrm{Pi}$ is an advancement of single-board PCs created in the United Kingdom by the Raspberry Pi Foundation. Peripherals are excluded with the Raspberry Pi.

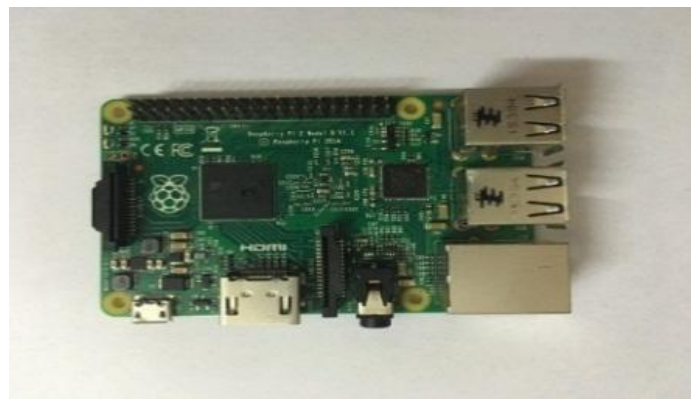

Fig 3: Front face Raspberry Pi

Raspberry Pi have been released some years ago. The first Raspberry Pi 1 Model was released in February 2012. It was followed by a Model A. In 2014, the foundation had released a board with an improved layout in Raspberry Pi 1 Model B+. These sheets are around Mastercard evaluated and address the standard mainline shape compute. Upgraded $\mathrm{A}+$ and $\mathrm{B}+$ models were released after one year. A "procedure module" was released in April 2014 for introduced applications, and a Raspberry Pi Zero with better size. The Raspberry Pi 2 which included more RAM was released in February 2015 Raspberry Pi 3 Model B released in February 2016, is bundled with on-board Wi-Fi, Bluetooth and USB boot capabilities. As of January 2017, Raspberry Pi 3 Model B is the most forward mainline Raspberry Pi. Raspberry Pi sheets are assessed between US\$5-35. Beginning at 28 February 2017, the Raspberry Pi Zero W was impelled

\section{Raspberry PI Development Board}

The Raspberry $\mathrm{Pi}$ is a single board computer developed in the UK by the Raspberry Pi Foundation with the motive of promoting the knowledge of basic computer science in schools. The Raspberry Pi is manufactured in two board configurations.

The Raspberry Pi utilizes a Broadcom framework on a chip and it was initially sent with 256 megabytes of RAM, later moved up to $512 \mathrm{MB}$. It does exclude an implicit hard plate or strong state drive, however it utilizes a SDcard for booting and relentless stockpiling. The Foundation gives Debian and Arch Linux ARM conveyances for download. Instruments are accessible for Python as the primary programming dialect. 


\section{Additional Hardware used}

- Power supply

- Hotspot creator

- Wi-Fi receiver

- $\quad$ PIR Sensor

- Female jumper wire

- $\quad$ Raspberry pi camera

\section{PROPOSED ALGORITHM}

Open main interface in mobile phone or computer Connect Raspberry Pi through Wi-Fi internet

Provide separate external power supply for Raspberry Pi Board and available appliances.

Start

Step 1: PIR sensor start sensing the motion

if motion sensed by the PIR sensor

then

$$
\text { Raspberry Pi send an email to the registered mail id }
$$$$
\text { Else }
$$

Keep sensing

End if condition

Step 2: For security purpose camera capture the image

if Camera read any person or object.

then

Image Captured by Camera.

else

\section{Keep reading}

End if condition

Step 3:To operate any available appliance through the given buttons in the interface

a) Click the Light $\mathrm{ON}$ switch through device to activate Light

If we give the correct command

Then

Light is ON

Else

Light is set to be in Standby Mode or OFF Mode Fan

b) Click the Fan ON switch through device to activate

if we give the correct command

Then

Fan is ON

Else

Fan is to be set in Standby mode or OFF Mode.

c) Click the AC ON switch through device to activate AC

if we give the correct command

Then

$$
\mathrm{AC} \text { is } \mathrm{ON}
$$

Else

AC is to be set in Standby mode or OFF Mode.

Step 4: To operate all available appliances through the Master switch given in main interface

Click on the Turn on Master switch by device

If we give the correct command

Then

$$
\text { All appliances turn } \mathrm{ON}
$$

else

All appliances are set to be in Standby or OFF Mode.

End of process

\section{FLOW CHART}

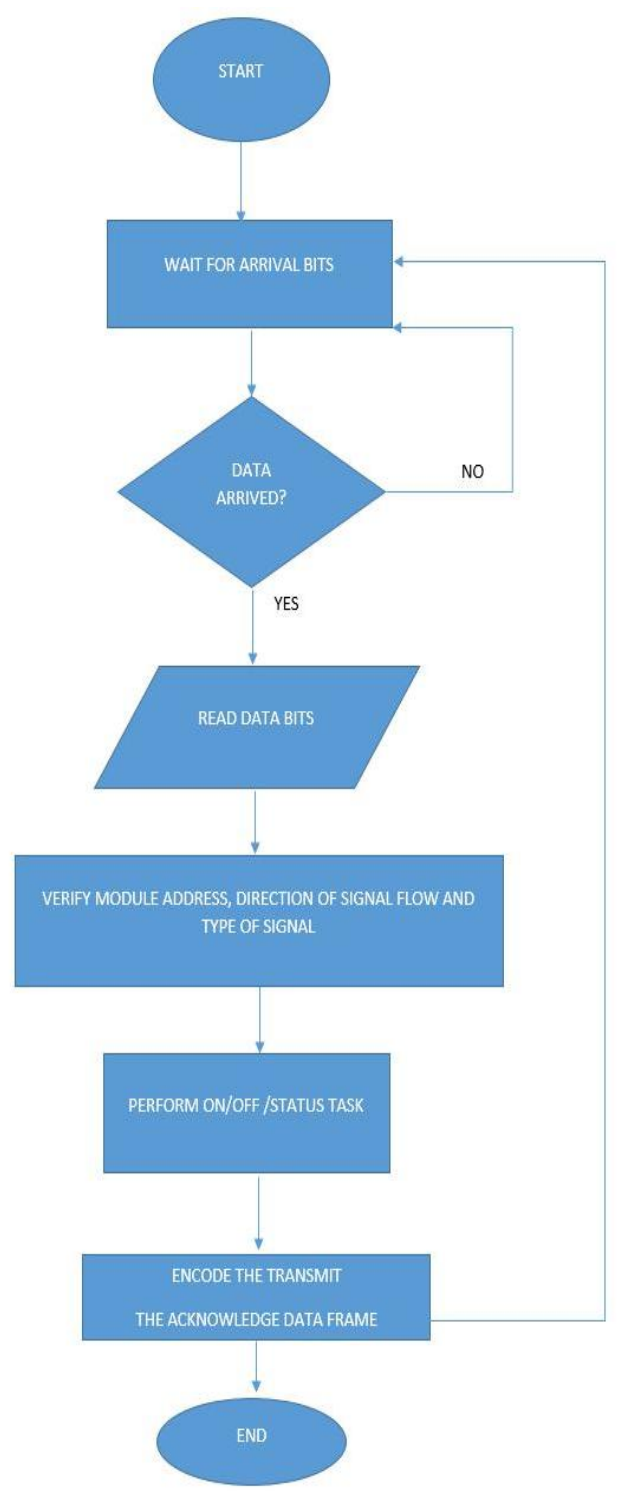




\section{IMPLEMENTATION}

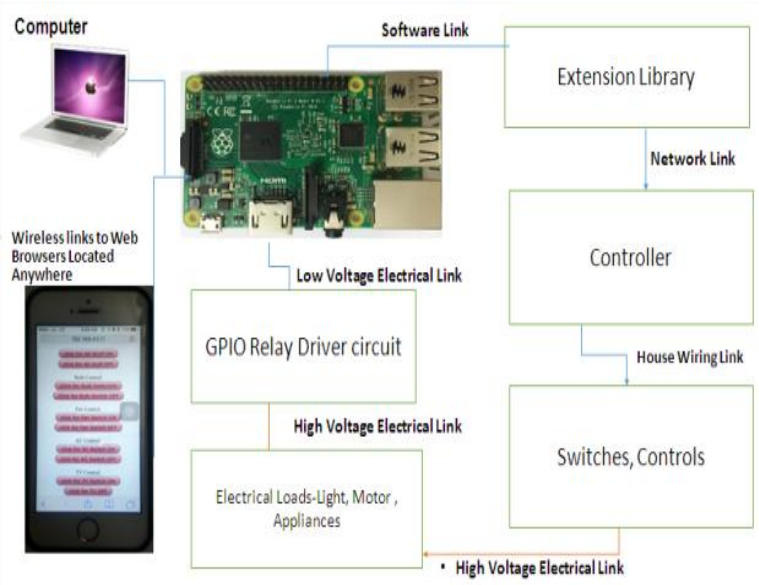

Fig 4: Overall Architecture

\subsection{Interface of proposed method}

In this figure we show the main interface of our working model. We can use this interface in any electronic device (that easily access internet) through given static IP address. As shown in the figure that we are providing controls for four appliances i.e, Bulb, Fan, AC and TV. We can change these controls or add more appliances upto 23 based on our requirement. Along with given appliances we are providing one master control to operate all the available appliances through a single button.

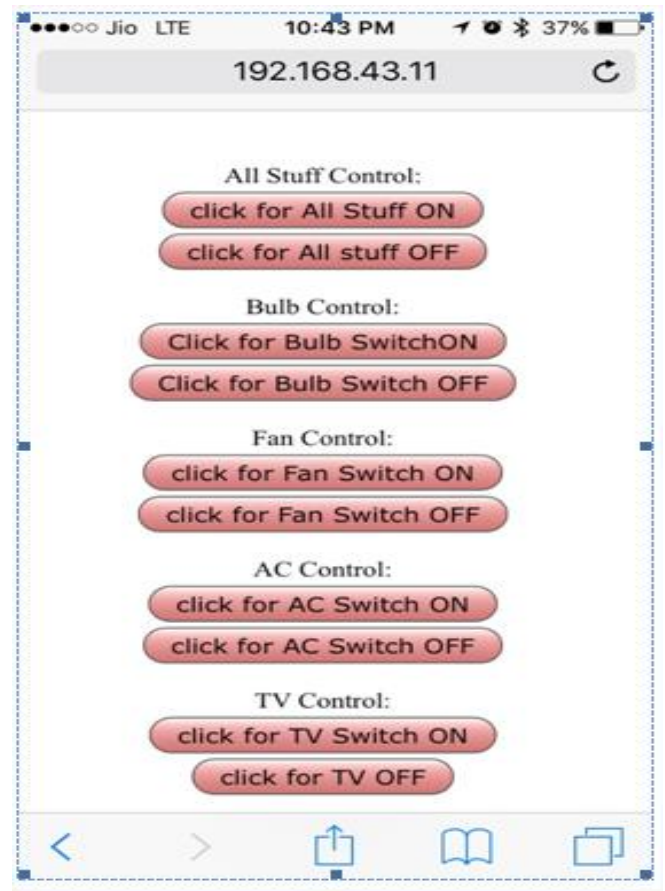

Fig 5: Main interface

\subsection{Prototype of proposed model in standby mode}

This figure shows the actual prototype of our model in which we place three sample appliances to show the working state of our proposed model. Initially we place all appliances in standby mode.

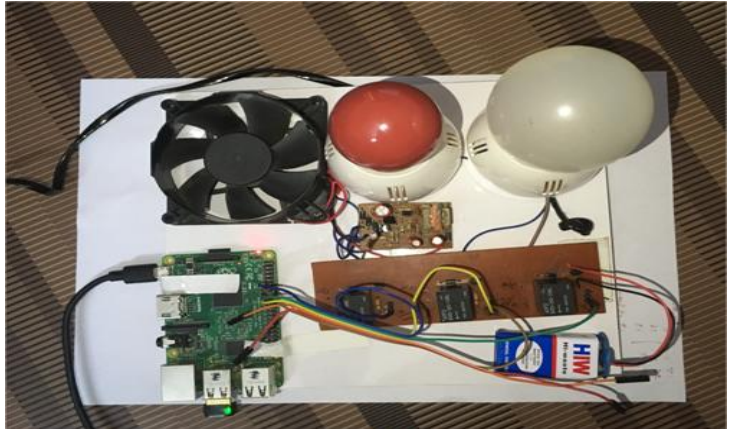

Fig 5: Prototype of proposed method in standby mode

\subsection{Prototype with all appliances in Active MODE}

In this figure we show the prototype model while we set al the appliances in the active state. It means at this moment all connected appliances are working by getting the command from master switch available on our main interface. This feature is used when we want to run all available devices simultaneously.

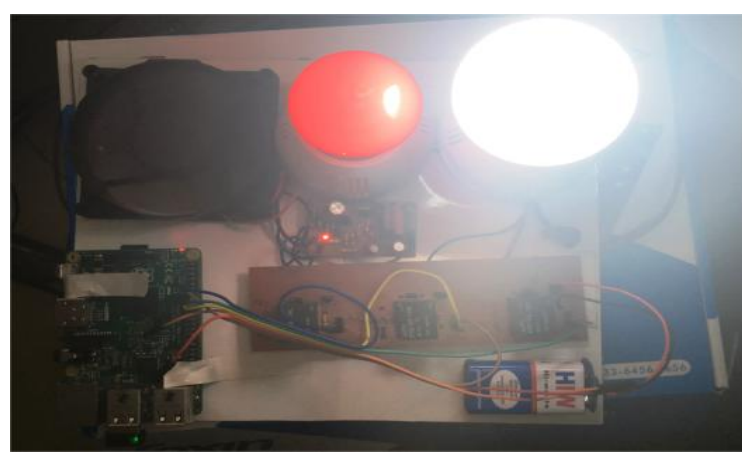

Fig 6: Prototype model with all appliances in Active mode

\section{RESULT AND PERFORMANCE ANALYSIS}

\subsection{Performance of Conventional method}

In this graph we show the performance of the conventional method based on three parameters that are time, temperature and life.

Table 1: Performance result of conventional method in Bread Board

\begin{tabular}{|c|c|c|c|}
\hline S. No. & Time & Temperature & Life \\
\hline 0 & 8 & 15 & 18 \\
\hline 1 & 10 & 16 & 17.5 \\
\hline 2 & 12 & 18 & 17 \\
\hline 3 & 14 & 19 & 16.5 \\
\hline 4 & 16 & 20 & 16 \\
\hline 5 & 18 & 21 & 15 \\
\hline 6 & 20 & 22 & 14 \\
\hline 7 & 22 & 23 & 13 \\
\hline 8 & 24 & 28 & 12 \\
\hline
\end{tabular}




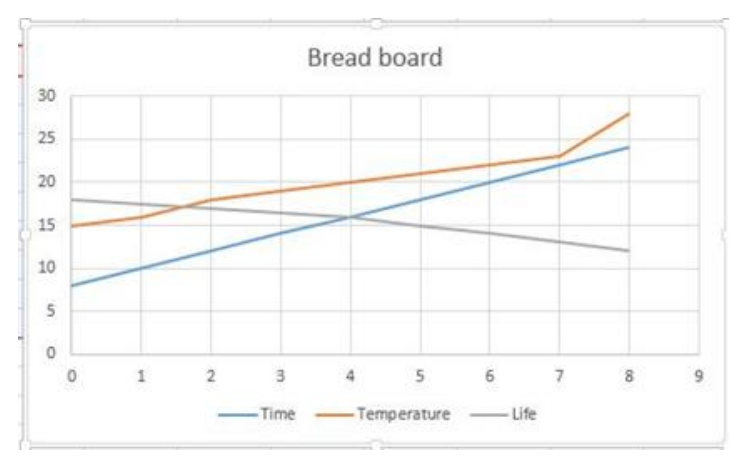

Fig 7::Performance graph of conventional method in Bread Board

\subsection{Performance of proposed method}

The following data shows the performance of the proposed method in PCB based on three parameters that are Time, Temperature and life.

Table 2: Performance result of proposed method in PC

\begin{tabular}{|c|c|c|c|}
\hline S. No. & Time & Temperature & Life \\
\hline 0 & 8 & 15 & 36 \\
\hline 1 & 10 & 16 & 34 \\
\hline 2 & 12 & 18 & 31 \\
\hline 3 & 14 & 19 & 30 \\
\hline 4 & 16 & 20 & 28 \\
\hline 5 & 18 & 21 & 26 \\
\hline 6 & 20 & 22 & 24 \\
\hline 7 & 22 & 23 & 22 \\
\hline 8 & 24 & 28 & 18 \\
\hline
\end{tabular}

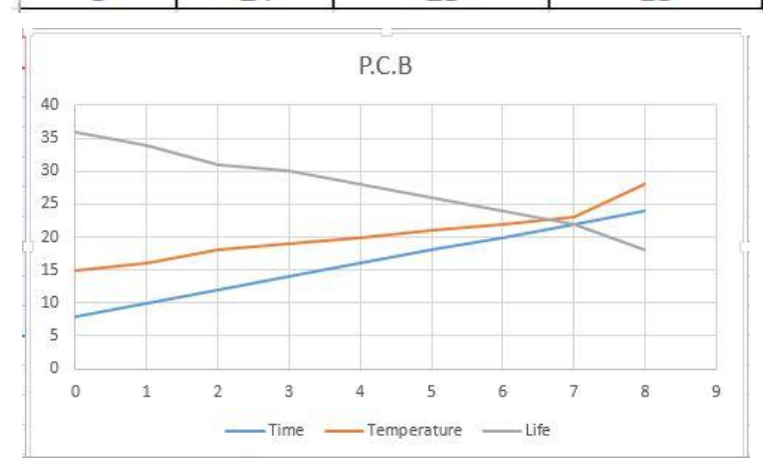

Fig 8: Performance graph of proposed method in PCB

\subsection{Comparison between Raspberry Pi 1} and Pi 2

In the following results we show the comparison of $\mathrm{Pi} 1$ hardware (used in conventional method) and $\mathrm{Pi} 2$ hardware(used in our proposed method). This comparison has been done on various factors based on hardware attributes like USB, Storage, Ethernet, SDRAM, Processor, Video.

Table 3: Comparison result of Pi 1 and $\mathrm{Pi} 2$

\begin{tabular}{|c|c|c|}
\hline Attributes & PI 1 & PI 2 \\
\hline USB & 2 & 4 \\
\hline Storage & 16 & 64 \\
\hline Ethernet & 100 & 100 \\
\hline SdRam & 512 & 1024 \\
\hline Processor & 900 & 1000 \\
\hline Video & 480 & 1080 \\
\hline
\end{tabular}

PI 1, PI 2

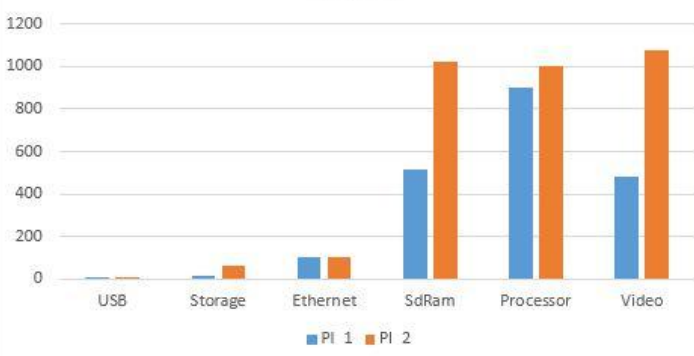

Fig 8: Comparison graph of $\mathrm{Pi} 1$ and $\mathrm{Pi}$

\subsection{Comparison of Conventional and Proposed Methods}

In this figure we show the comparison of conventional and proposed method together based on various factors like Time, Temperature, life of conventional method and life of proposed method.

This graph depicts that the our proposed method is better than conventional method in all manner.

Table 4: Comparison data of conventional and proposed methods

\begin{tabular}{|c|c|c|c|r|}
\hline S. No. & Time & Temperature & B.life & P.life \\
\hline 0 & 8 & 15 & 18 & 36 \\
\hline 1 & 10 & 16 & 17.5 & 34 \\
\hline 2 & 12 & 18 & 17 & 31 \\
\hline 3 & 14 & 19 & 16.5 & 30 \\
\hline 4 & 16 & 20 & 16 & 28 \\
\hline 5 & 18 & 21 & 15 & 26 \\
\hline 6 & 20 & 22 & 14 & 24 \\
\hline 7 & 22 & 23 & 13 & 22 \\
\hline 8 & 24 & 28 & 12 & 18 \\
\hline
\end{tabular}

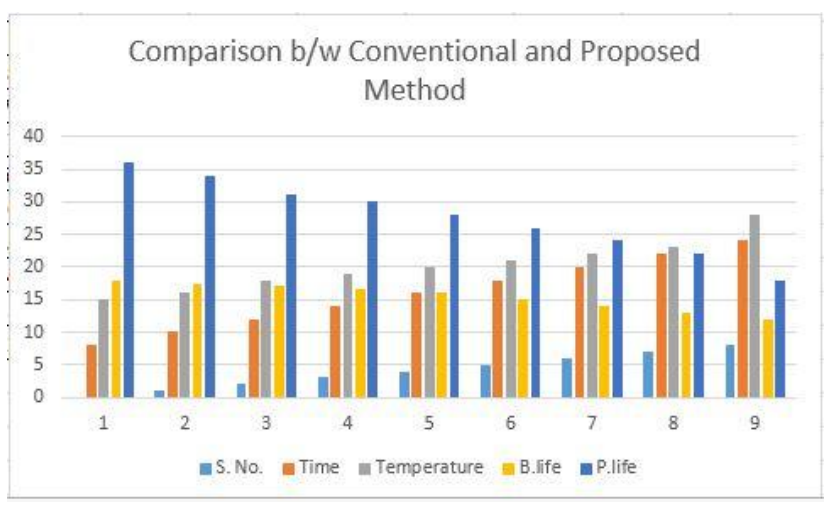

Fig 8: Comparison graph of conventional and proposed methods

\subsection{Comparison of Conventional and Proposed methods based on various features}

In the given table we give the comparison of both the methods based on the availability of mentioned features. As the data shown in following table we can say that the proposed method will overcome all the limitations of the conventional method and provide all possible features as per our requirement. 
Table 5: Comparison table of Conventional and Proposed method

\begin{tabular}{|c|l|l|l|}
\hline S.NO. & Attributes & Conventional & Proposed \\
\hline 1 & Ethernet & Yes & Yes \\
\hline 2 & Wi-Fi & No & Yes \\
\hline 3 & Recording & No & Yes \\
\hline 4 & Mail Notification & No & Yes \\
\hline 5 & PIRSensed & No & Yes \\
\hline 6 & Appliances ON/Off & Yes & Yes \\
\hline
\end{tabular}

\section{CONCLUSION}

These types of home automation frameworks are required in the situation when human forgot to turn off the home appliances when there is no utilization. In this way they are helpful to control our home appliances through our smart phone from anywhere anytime and therefore it is also useful to cut unwanted electricity bills as well as it increases the life of our appliances as they are used only when they are required.

\section{FUTURE SCOPE}

The future of Home Automation is very vast. We can add many more new features in it in future to make it more efficient to fulfill user's requirement. Some features are given below.

1. Home Security system goes wireless

2. It can be operated through voice command

3. Temperature sensors

4. User can add new appliances by their own in a given interface without any external support.

\section{REFERENCES}

[1] P Bhaskar Rao, S.K.Uma, "Raspberry Pi Home Automation with Wireless sensors using smart phone" International Journal of Computer Science and Mobile Computing, vol.4, issue.5, pg 797-803, May 2015.

[2] P Pavan Kumar, G Tirumala Vasu, "Home Automation \& Security System Using Arduino Android ADK" International Journal of Emerging Trends in Engineering Research, Volume.3, No.6, pp:190-194, 2015.

[3] Zekeriyakeskin, YunusEmrekocaturk, okanBingol, kubilayTasdelen, "Web-based smart home automation: PLC controlled implementation", vol 11,NO 3,2014

[4] S.Banjamin Arul, "Wireless Home Automation System Using Zigbee" International Journal of Scientific \&
Engineering Research, Volume.5, Issue.12, December 2014.

[5] Jayashri Bengali, ArvindShaligram, "Energy efficient Smart home based on Wireless Sensor Network using LabVIEW" American Journal of Engineering Research, Volume 2, Issue 12, pp 409-413. 2013

[6] HariCharanTadimeti, ManasPulipati, “Overview of Automation Systems and Home Appliances Control using PC and Microcontroller", Volume 2 Issue 4, April 2013

[7] Sirsath N.S, Dhole P.S, Mohire N.P, Naik S.C, Ratnaparkhi N.S, "Home Automation Using Cloud Network and Mobile Devices" ITSI Transactions on Electrical \& Electronics Engineering, Volume-1, Issue-2, 2013.

[8] Basma M. Mohammad El-Basioni, Sherine M. Abd Elkader and Mahmoud AbdelmonimFakhreldin"Smart Home Design using Wireless Sensor Network and Biometric Technologies", International Journal of Application or Innovation in Engineering \& Management, Volume 2, Issue 3, March 2013

[9] Prof. M. B. Salunke, Darshan Sonar, NileshDengle ,SachinKangude, DattatrayaGawade, "Home Automation Using Cloud Computing and Mobile Devices", Vol. 3, Issue 2, pp: 35-37, Feb. 2013

[10] Volume 6, Issue 1 (May. - Jun. 2013), PP 65-75 www.iosrjournals.org www.iosrjournal.orgVoice Recognition Wireless Home Automation System Based OnZigbeeDhawan S. Thakur1 and Aditi Sharma2. Eternal University, Himachal Pradesh, India

[11] R. A. Ramlee, M. H. Leong, R. S. S. Singh, M. M. Ismail, M. A. Othman, H. A. Sulaiman, et al., "Bluetooth remote Home Automation System Using Android Application," The International Journal of Engineering And Science, vol. 2, pp. 149-153, 11, January 2013.

[12] A. ElShafee and K. A. Hamed, "Design and Implementation of a Wi-Fi Based Home Automation System," World Academy of Science, Engineering and Technology, vol. 68, pp. 2177-2180, 2012.

[13] Sajidullah S. Khan, Anuja Khoduskar, Dr. N.A,Koli,"Home automation system", IJAET/Vol.II/April-June, 2011/12. 